\author{
Katharina Marten \\ David Milne \\ Katerina M. Antoniou \\ Andrew G. Nicholson \\ Rachel C. Tennant \\ Trevor T. Hansel \\ Athol U. Wells \\ David M. Hansell
}

\title{
Non-specific interstitial pneumonia in cigarette smokers: a CT study
}

Received: 26 August 2008

Accepted: 27 December 2008

Published online: 13 February 2009

(C) The Author(s) 2009.

This article is published with open access at

Springerlink.com

K. Marten $(\bowtie)$

Department of Radiology,

Georg August University of Göttingen,

Robert-Koch-Str. 40,

37075 Göttingen, Germany

e-mail: kmarten@med.uni-goettingen.

de

Tel.: +49-551-398965

\section{Milne}

Department of Radiology,

Green Lane Hospital,

Auckland, New Zealand

\section{K. M. Antoniou}

Department of Thoracic Medicine,

University of Crete,

Heraklion, Crete, Greece

A. G. Nicholson

Department of Histopathology,

Royal Brompton Hospital,

London, UK

\author{
R. C. Tennant · A. U. Wells \\ Interstitial Lung Disease Unit, \\ Royal Brompton Hospital, \\ London, UK \\ T. T. Hansel \\ Clinical Trials Unit, \\ Royal Brompton Hospital, \\ London, UK \\ D. M. Hansell \\ Department of Radiology, \\ Royal Brompton Hospital, \\ London, UK
}

\begin{abstract}
The goal of this study was to seek indirect evidence that smoking is an aetiological factor in some patients with non-specific interstitial pneumonia (NSIP). Ten current and eight exsmokers with NSIP were compared to controls including 137 current smokers with no known interstitial lung disease and 11 non-smokers with NSIP. Prevalence and extent of emphysema in 18 smokers with NSIP were compared with subjects meeting GOLD criteria for chronic obstructive pulmonary disease (COPD; group A; $n=34$ ) and
\end{abstract}

healthy smokers (normal $\mathrm{FEV}_{1}$; group $\mathrm{B} ; n=103$ ), respectively. Emphysema was present in 14/18 (77.8\%) smokers with NSIP. Emphysema did not differ in prevalence between NSIP patients and group A controls $(25 / 34,73.5 \%)$, but was strikingly more prevalent in NSIP patients than in group $\mathrm{B}$ controls $(18 / 103,17.5 \%, P<0.0005)$. On multiple logistic regression, the likelihood of emphysema increased when NSIP was present $(\mathrm{OR}=18.8 ; 95 \% \mathrm{CI}=5.3-$ 66.3; $P<0.0005)$ and with increasing age $(\mathrm{OR}=1.04 ; 95 \% \mathrm{CI}=0.99-1.11$; $P=0.08$ ). Emphysema is as prevalent in smokers with NSIP as in smokers with COPD, and is strikingly more prevalent in these two groups than in healthy smoking controls. The association between NSIP and emphysema provides indirect support for a smoking pathogenesis hypothesis in some NSIP patients.

Keywords Nonspecific interstitial pneumonia $\cdot$ Pulmonary fibrosis . Emphysema $\cdot$ Cigarette smoking

\section{Introduction}

In recent years, cigarette smoking has been implicated in the development of various interstitial lung diseases: respiratory bronchiolitis-associated interstitial lung disease, desquamative interstitial pneumonia (DIP) and pulmonary Langerhans cell histiocytosis are now considered to represent different types of response to cigarette smoke exposure $[1,2]$. Cigarette smoking is believed to be a risk factor for the development of idiopathic pulmonary fibrosis, and in some individuals emphysema and pulmonary fibrosis coexist $[3,4]$. In a retrospective study of highresolution CT (HRCT) appearances in patients with biopsy-proven DIP, two out of six smokers showed HRCT features suggestive of fibrotic non-specific interstitial pneumonia (NSIP) rather than DIP [5]. This has led to 
the hypothesis that a subgroup of cigarette smokers may develop a pattern of fibrotic NSIP.

However, support for this hypothesis can come only from indirect evidence, as it is not practicable to undertake a prospective longitudinal evaluation of smokers and nonsmokers, necessarily lasting for many years, to determine whether NSIP, a relatively rare disorder, develops selectively in some smokers. Support for the idea of a smokingrelated pathogenesis for NSIP could be established from two sources. First, an association between NSIP and known smoking-related damage, such as centrilobular emphysema, could suggest that NSIP and emphysema have common pathogenetic features. Secondly, differences in the HRCT appearances of NSIP itself in smokers and nonsmokers would support the hypothesis that smoking status is linked to pathogenesis in a subset of patients. This retrospective study was constructed to determine whether such evidence exists.

The prevalence of pulmonary emphysema in NSIP was compared to that in smoking control subjects enrolled in a smoking cessation study undergoing limited HRCT examination and subdivided into subjects meeting pulmonary function criteria for chronic obstructive pulmonary disease (COPD) and those with normal $\mathrm{FEV}_{1}$.

Some of the results of these studies have been previously reported in the form of an abstract [6].

\section{Materials and methods}

\section{NSIP patients}

Twenty-nine consecutive patients with fibrotic NSIP (diagnosed on open or video-assisted thoracoscopic biopsy, with all biopsies reviewed by the same two histopathologists) and concurrent HRCT (within 12 months, median 2 months) between January 1990 and June 2004 were studied. There were 19 men and 10 women, median age was 53 years, range 31-68 years (mean and SD given in Table 1). The individuals were classified as current smokers, ex-smokers (a minimum of one cigarette a day for a minimum of 1 year, stopping at least 6 months before presentation), or those who had never smoked (nonsmokers). Patients with an environmental exposure to a fibrogenic agent or with a connective tissue disease were not included in the study population.
Smoking control population

A cohort of 137 current smokers who had undergone a limited HRCT of the lungs comprised the control group. These subjects had been enrolled in a smoking cessation study at our institution between May 2002 and June 2004. Approval was obtained from our institutional ethics committee for the smoking cessation trial, and all patients gave written informed consent after the purpose and nature of the CT examination were explained. Enrolment in the smoking cessation study required either that patients met GOLD criteria for COPD [7] (group A, $n=34)$ or that $\mathrm{FEV}_{1}$ levels were normal, i.e. $>90 \%$ of predicted (group B, $n=103$ ).

Smoking controls were sub-categorised as "healthy" smokers or as COPD controls. COPD controls $(n=34)$ fulfilled GOLD group II or III criteria, defined on spirometry by a post-bronchodilator $\mathrm{FEV}_{1}$ of $30-80 \%$ predicted following the inhalation of salbutamol $(400 \mu \mathrm{g}$ via MDI) [7]. The pre-bronchodilator forced expiratory ratio (FER) was $<70 \%$, and reversibility to bronchodilator was less than $12 \%$ or $200 \mathrm{ml}$. Subjects in this group were aged 40-80 years, current smokers of at least five cigarettes per day with a greater than 10-pack-year history and no history of atopy, asthma, or other respiratory disease. Individuals with a history of malignancy in the past 5 years, excepting basal-cell skin carcinoma, were excluded. Use of inhaled corticosteroids, theophylline, long-acting $\mathrm{b}$ agonists and non-steroidal anti-inflammatory drugs was not permitted. Permitted non-anti-inflammatory therapy included medication for ischemic heart disease and hypertension.

"Healthy" smokers (group B, $n=103$ ) were defined on spirometry by $\mathrm{FEV}_{1}>90 \%$ predicted and FER of $>70 \%$. They were matched with the COPD subjects in terms of age, sex, and smoking history. Exclusion criteria included a history of atopy or any respiratory disease including chronic simple bronchitis. Respiratory medication was not permitted during the duration of the study. All smokers were actively helped in efforts to stop smoking.

\section{Pulmonary function tests}

In all patients, pulmonary function tests, performed within 1 month of CT, included $\mathrm{FEV}_{1}, \mathrm{FVC}$, and $\mathrm{DL}_{\mathrm{CO}}$ corrected

Table 1 Demographic data and pack-year smoking histories compared between NSIP patients and the smoking control groups

\begin{tabular}{llll}
\hline & $\begin{array}{l}\text { NSIP patients (current } \\
\text { and ex-smokers) }(n=18)\end{array}$ & $\begin{array}{l}\text { Group A (meeting criteria } \\
\text { for COPD) }(n=34)\end{array}$ & $\begin{array}{l}\text { Group B (FEV } \\
\% \text { of predicted) }(n=103)\end{array}$ \\
\hline Age (mean \pm SD) & $52.8 \pm 7.7 \mathrm{a}^{* *}$ & $59.4 \pm 10.2 \mathrm{a}^{* *}$ & $\begin{array}{l}53.6 \pm 8.8 \\
42: 56 \mathrm{a}^{*}\end{array}$ \\
Male/female & $13: 5 \mathrm{a}^{*}$ & $17: 17$ & $\begin{array}{l}44 \mathrm{a}^{* *}(10-195) \\
\text { Pack-year smoking history (median, range) }\end{array}$ \\
\hline
\end{tabular}

Entries in a row marked with the same letters are statistically different. $* P=0.03, * * P<0.01, * * * P=0.001, * * * * P<0.0001$ 
for haemoglobin concentration, expressed as percentages of the predicted normal values.

\section{High-resolution CT}

Computed tomography was performed on a four-channel multidetector Somatom Volume Zoom CT system (Siemens, Erlangen, Germany) using $1.25-\mathrm{mm}$ collimation and a gantry rotation time of $0.8 \mathrm{~ms}$, or on an electron beam CT system (Imatron, San Francisco, CA) using 1.5-mm collimation and an acquisition time of $100 \mathrm{~ms}$. Lung image datasets were reconstructed with a high spatial frequency algorithm and viewed on work stations at window settings appropriate for the lung parenchyma.

The asymptomatic smokers enrolled in the smoking cessation trial had a limited high-resolution CT consisting of four interspaced 1.25-mm sections: (1) at the mid aortic arch, (2) at the main carina, (3) equally spaced between the main carina and the dome of the higher hemi-diaphragm and (4) $1 \mathrm{~cm}$ above the dome of the higher hemidiaphragm. Patients with NSIP had a high-resolution CT of the entire lungs, with sections obtained at $10-\mathrm{mm}$ intervals in the supine position in full inspiration. From these CT examinations, four sections corresponding to those acquired in the asymptomatic smoking controls were retrospectively selected and stored as a separate series.

All CT examinations were independently reviewed by two experienced observers who were unaware of clinical information or biopsy findings. All potential smokingrelated abnormalities were scored. The observers were unaware of the study design and specifically that the prevalence of emphysema was a primary focus of the study.

In NSIP patients and smoking controls, the presence or absence of emphysema was recorded; disagreement was resolved by consensus. Subsequently, in NSIP patients, the five CT sections were scored to the nearest $5 \%$ for the extent of lung with interstitial disease. An estimation was made to the nearest $5 \%$ (summing to $100 \%$ ) of the contribution made by individual patterns as defined in the Fleischner Society glossary [8] (ground-glass opacification with traction bronchiectasis, pure ground-glass opacification, honeycombing, a reticular pattern not otherwise specified, nodules, lobules of decreased attenuation likely to represent air-trapping, a pattern resembling the appearance of pulmonary Langerhans cell histiocytosis, "crazy paving" pattern and consolidation). The extents of individual patterns (examples shown in Figs. 1, 2 and 3) were derived, and the mean values for the two observers were analysed.

\section{Data analysis}

Analyses were performed with STATA software (Stata data analysis software; Computing Resource Center, Santa
Monica, CA). A $P$-value of $<0.05$ was taken to indicate statistical significance. Interobserver variation was quantified using Kappa statistics or, for continuous variables, the single determination standard deviation. Analyses were undertaken to examine the following questions.

1) Does the prevalence of emphysema differ between patients with NSIP and control smoking subjects in group A (individuals meeting GOLD criteria for COPD)? This question was evaluated using Fisher's exact test, and then using multiple logistic regression, adjusting for age, gender and the pack-year smoking history.

2) Does the prevalence of emphysema differ between patients with NSIP and control smoking subjects in group $\mathrm{B}$ (those with normal $\mathrm{FEV}_{1}$ levels)? This question was examined (a) with the retention of both ex- and current smokers with NSIP and (b) with the exclusion of ex-smokers with NSIP (in order to exclude bias due to a "healthy-smoker effect"). Subgroup comparisons were made using Fisher's exact test, and then using multiple logistic regression to adjust for age, gender and the pack-year smoking history.

3) Does the presence or extent of HRCT features of interstitial lung disease differ between non-smokers with NSIP and ex/current smokers with NSIP? For HRCT features occurring in a minority of patients, prevalence was compared using Fisher's exact test, with further evaluation of significant trends using the Kruskal-Wallis test (examining prevalence differences across non-smokers, ex-smokers and current smokers). For HRCT features present in the majority of patients, the extent of patterns was compared between subgroups using Wilcoxon's rank sum test.

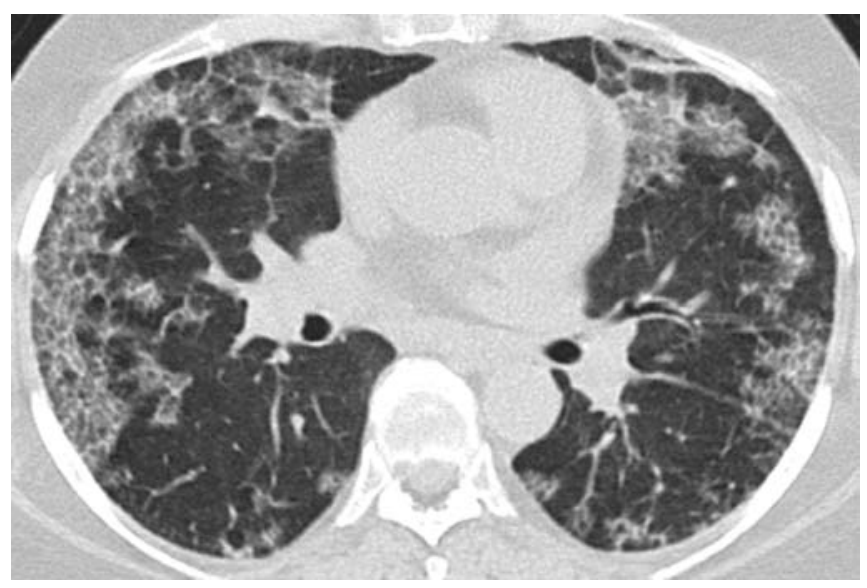

Fig. 1 Thin-section CT of a 44-year-old female non-smoker with NSIP showing widespread diffuse ground-glass opacification with some thickening of the interlobular septa (crazy-paving pattern). Total extent of interstitial disease was scored as 35\% (40\% assigned as ground-glass opacification without traction bronchiectasis and $60 \%$ as crazy-paving pattern) 


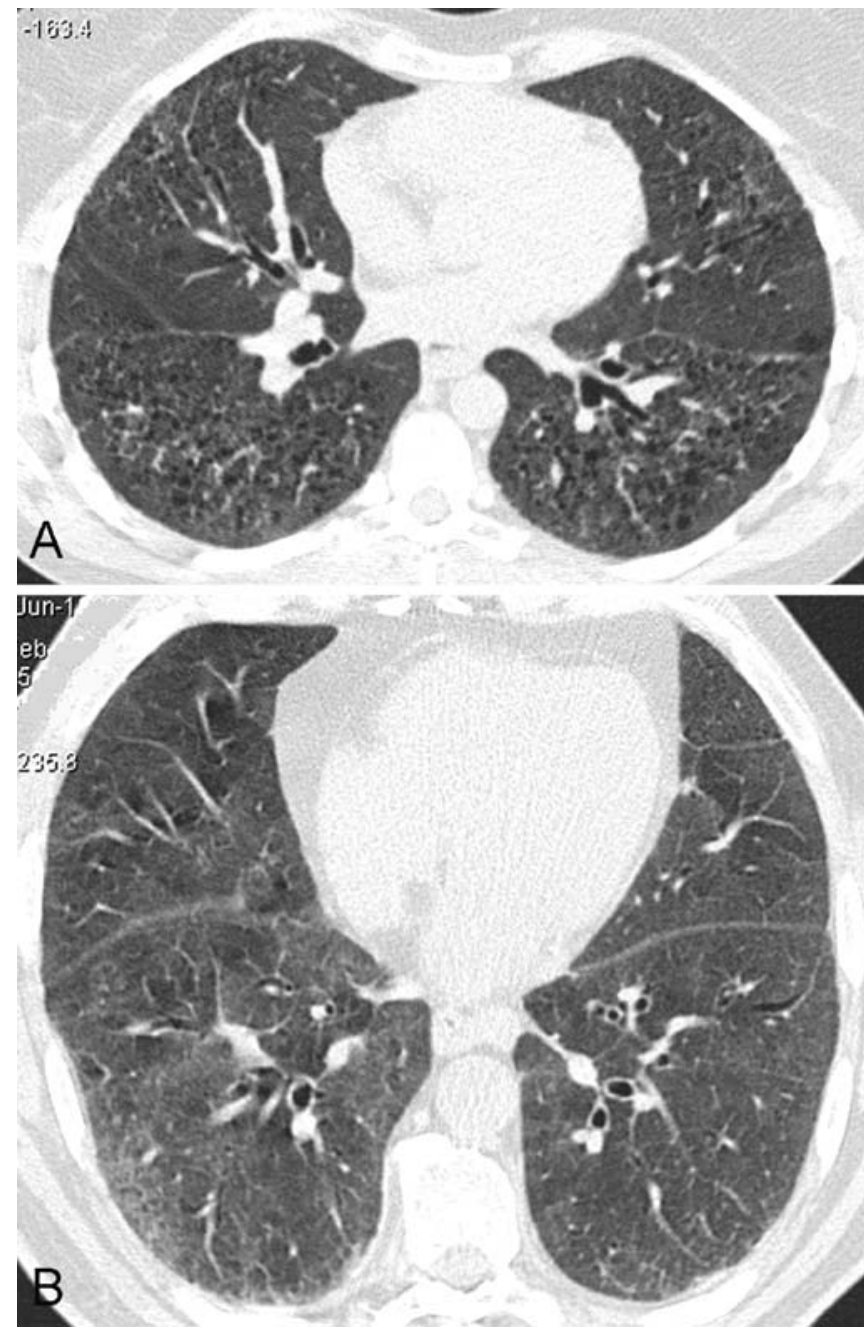

Fig. 2a, b Thin-section CT of two current smokers with NSIP. a A 43-year-old woman with a 15-pack-year smoking history displaying diffuse ground-glass opacification with superimposed centrilobular emphysema. Total extent of interstitial disease was scored as $95 \%$ ( $20 \%$ attributed to emphysema, and $80 \%$ to ground-glass opacification without traction bronchiectasis). b A 60 -year-old man with a 45-pack-year smoking history with diffuse ground-glass opacification with traction bronchiectasis (extent scored as 100\%)

\section{Results}

Demographic details and pack-year smoking histories

As shown in the Table 1, NSIP smoking patients were younger than group A controls and had a much lower packyear smoking history (median 22.5, range 3-70 versus median 60, range $25-190, P<0.0001$ ). NSIP patients had a significantly lower pack-year smoking history and were more likely to be male than group B controls. Review of case records revealed that at presentation all NSIP patients had a history of exertional dyspnea, and basal inspiratory crackles at clinical examination. None had a prior diagnosis of COPD. The median duration of symptoms was 24 months (range 4-120 months). Eight patients were exsmokers (median pack-years: 10, range: 5-30), and ten patients were current smokers (median pack-years: 46, range: $10-70)$.

Prevalence of emphysema in NSIP patients and group A (smokers with COPD)

The prevalence of emphysema did not differ between NSIP patients $(14 / 18,77.8 \%)$ and group A control subjects $(25 / 34,73.5 \%), P=0.74$. On logistic regression, there was no significant or marginal difference in the prevalence of emphysema between the two sub-groups after adjustment for age, gender and pack-year smoking history.

Prevalence of emphysema in NSIP patients and in group B (healthy smokers)

Emphysema was present in 14 out of 18 NSIP cases (77.8\%) compared to 18 of 103 group B control subjects $(17.5 \%), P<0.0005$ (Fig. 1). On logistic regression, the presence of emphysema was associated with a diagnosis of NSIP [odds ratio $18.8 ; 95 \%$ confidence interval $(95 \% \mathrm{CI})$ 5.3-66.2; $P<0.0005]$ and with increasing age (odds ratio 1.04 ; $95 \%$ CI0.99-1.11; $P=0.08$ ). Gender and the packyear smoking history were not separately linked to the presence of emphysema.

With the exclusion of 9 ex-smokers with NSIP, the trends were unchanged. Emphysema (present in 9 of 10 current smokers with NSIP) was more prevalent than in group B control subjects $(18$ of 103$)(P<0.0005)$. On



Fig. 3 Thin-section CT of a current smoker with NSIP (60-year-old woman with a 39-pack-year smoking history). Total extent of interstitial disease was scored as $40 \%(50 \%$ attributed to a reticular pattern, $30 \%$ to ground-glass opacification with traction bronchiectasis, and $20 \%$ to ground-glass opacification without traction bronchiectasis) 
logistic regression, the presence of emphysema was again associated with a diagnosis of NSIP (odds ratio $57.4 ; 95 \%$ CI6.3-516.4; $P<0.0005$ ) and with increasing age (odds ratio 1.06 per year of increase; $95 \% \mathrm{CI} 1.00-1.13 ; P=0.04$ ). Gender and the pack-year smoking history were not separately linked to the presence of emphysema.

\section{CT extent of emphysema compared} between sub-groups

Inter-observer agreement for the presence or absence of emphysema on HRCT, combining NSIP and control subjects, was excellent $(K=0.87)$. The single-determination standard deviation for the extent of emphysema on CT was $4.6 \%$. The extent of emphysema on CT did not differ between patients with NSIP (median $11.2 \%$, range 0$51.7 \%$ ) and group A COPD control subjects (median 4.3\%, range $0-74.4 \%), P=0.39$, and this finding was unchanged $(P=0.50)$ when patients with no emphysema were excluded. Emphysema was more extensive in NSIP patients than in group $\mathrm{B}$ healthy smoking control subjects (median 0, range $0-18.5$ ), $P<0.0001$. This finding remained significant, $P<0.005$, when patients with no emphysema were excluded.

CT interstitial patterns compared between smokers and non-smokers with NSIP

The single-determination SDs for the CT extent of groundglass opacification with traction bronchiectasis, pure ground-glass opacification, and a reticular pattern were 5.4, 10.7, and 13.4\%, respectively. Agreement on the presence or absence of a crazy-paving pattern was fair to moderate $(K=0.39)$. Nodules, a pattern consistent with Langerhans cell histiocytosis, significant honeycombing, lobules of decreased attenuation or consolidation occurred in fewer than five NSIP patients, and these patterns were not analysed further.

The total extent of interstitial disease, the extent of honeycombing, the extent of ground glass (with or without traction bronchiectasis) and the extent of a reticular pattern did not differ significantly between non-smokers and current/ex-smokers. On logistic regression, the presence of emphysema was not linked to the extent of any of these HRCT patterns. A crazy-paving pattern was present in 7 of 10 non-smokers, 2 of 8 ex-smokers, but in none of 11 current smokers $(P<0.01$, chi-squared test for trend).

Functional significance of emphysema in smokers and non-smokers with NSIP

The presence of emphysema on HRCT was associated with higher FVC levels $(88.6 \pm 25.3$ versus $69.4 \pm 20.6 \%, P=$
$0.03)$, despite slightly lower $\mathrm{DL}_{\mathrm{CO}}$ levels $(42.8 \pm 12.8$ versus $49.1 \pm 18.7 \%, P=0.30$ ). On multivariate analysis, FVC levels diminished significantly with increasingly extensive interstitial lung disease [regression coefficient $(\mathrm{RC})=-0.96,95 \% \mathrm{CI}=-1.8$ to $-0.1, P=0.03]$ and increased marginally when emphysema was present $(\mathrm{RC}=$ $15.5 ; 95 \% \quad \mathrm{CI}=-1.1$ to $32.2 ; P=0.07)$. $\mathrm{DL}_{\mathrm{CO}}$ levels diminished significantly with increasingly extensive interstitial lung disease $(\mathrm{RC}=-0.69 ; 95 \% \mathrm{CI}=-1.3$ to $-0.1 ; P=$ 0.02 ) and marginally with the presence of emphysema $(\mathrm{RC}=-8.9 ; 95 \% \mathrm{CI}=-20.6$ to $2.8 ; P=0.13)$. The FVC-to$\mathrm{DL}_{\mathrm{CO}}$ ratio was higher in patients with emphysema (median 1.99, range 1.35-4.14) than in those without emphysema (median 1.43, range 1.15-3.46), $P<0.01$.

\section{Discussion}

We found a higher than expected prevalence of emphysema in cigarette smokers with NSIP, and our results support the concept that smoking may contribute to the pathogenesis of NSIP in some individuals.

The causative role of smoking in pulmonary fibrosis remains controversial. Specifically, relationships between cigarette smoking and the two commonest fibrosing idiopathic interstitial pneumonias, UIP and NSIP, have not been fully elucidated. Clinical studies that have sought to identify the relative risk of developing fibrosis in cigarette smokers are difficult to interpret, largely due to differences in the definition of the presence and type of interstitial fibrosis. Indeed, studies of subjects with idiopathic pulmonary fibrosis (IPF) have reported a varying prevalence of current or previous smokers $(41-83 \%)$ [916]. However, these studies predate the reclassification of IPF that clearly distinguishes between UIP and NSIP [17].

Possible pathogenetic mechanisms linking smoking with interstitial fibrosis include excessive oxidative stress [18, 19] and the modulation of oxidative stress by the upregulation of protease inhibitors (such as tissue inhibitor of metalloproteinases) [20] or a direct effect of smoking on the extracellular thiol/disulfide redox environment [21]. In IPF, it has recently been reported that severity-adjusted mortality is higher in current and former smokers than in life-long non-smokers, suggesting that smoking status is linked to the rapidity of progression of pulmonary fibrosis [22]. This effect may not be disease specific. A study by Craig et al. suggested that cigarette smokers may develop a pattern of fibrotic NSIP, either as a primary disorder or evolving from pre-existing DIP [5]. In this context, it is conceivable that in a subset of patients with a smoking history, macrophage accumulation of DIP recedes as time passes, leaving residual interstitial fibrosis in the form of NSIP.

Further indirect support for the hypothesis of a cigarette smoking-related pathogenesis in NSIP comes from the observation that HRCT morphologic features differ 
between non-smokers and current or former smokers. A crazy-paving pattern (the appearance of thickened interlobular septa superimposed on areas of ground-glass opacification) was frequent in non-smokers but was seldom present in ex- or current smokers and was never seen when significant smoking-related macrophage accumulation was present at biopsy. Crazy-paving was first described in alveolar proteinosis [23] and is now regarded as a nonspecific feature present in a variety of infectious, neoplastic and inhalational disorders [24]. Despite the statistical significance and novelty of the crazy-paving association, the low number of NSIP patients in the smoking subgroups demands that this observation be interpreted with caution. However, the finding of differences in HRCT patterns between smokers and non-smokers with NSIP indirectly supports the concept of smoking-related NSIP.

Two alternative explanations for the association between emphysema and NSIP merit consideration. A common genetic predilection might exist, but such a predilection is likely to relate to a pathogenetic mechanism that is common to the two diseases. Whether the linking trigger is genetic, environmental or a combination, our findings can be interpreted as justification for an increased focus on the candidate shared mechanisms discussed above. Furthermore, it is difficult to argue that the high prevalence of emphysema in NSIP in our study resulted from the disclosure of trivial emphysema by the presence of interstitial lung disease. Firstly, there was smoking-related centrilobular emphysema in all cases in lung regions not affected by NSIP (although there was also an admixture of the two processes in most patients). Secondly, the presence of emphysema across the whole NSIP cohort was associated with a clear effect on the FVC-to- $\mathrm{DL}_{\mathrm{CO}}$ ratio, indicative of overtly functionally significant disease.

The selection of control subjects is an important further consideration in a study of this type. Firstly, the CT examinations analysed were performed in consecutive attendees at a smoking cessation clinic and this may have introduced bias. However, a "healthy-smoker effect" [25] should mean that attendance at a smoking cessation clinic is associated with a greater likelihood of significant underlying lung damage; this would be consistent with the high prevalence of emphysema seen on HRCT in the control cohort. Thus, it appears highly unlikely that findings are spuriously positive because of an understatement of the prevalence of emphysema in smokers. More importantly, it was necessary to ensure that smoking controls and NSIP patients were comparable in having no prior diagnosis of smoking-related COPD. However, it could be argued that COPD might have become evident in some of the NSIP patients but for the development of pulmonary fibrosis lung disease, and this prompted us to re-evaluate associations in a combined analysis of NSIP patients and patients meeting GOLD criteria for a diagnosis of COPD. Strikingly, the prevalence of emphysema in these two groups was very similar.

A potentially important limitation in study design was that the HRCT scorers could not be blinded as to patient sub-group in determining the prevalence of emphysema due to the obvious presence of NSIP. To overcome this constraint, the primary purpose of the study (examining associations between NSIP and the presence of emphyse$\mathrm{ma}$ ) was not divulged to the scorers prior to the scoring of NSIP cases (which preceded the scoring of smoking controls). The high prevalence of emphysema in the smoking controls indicates that systematic underestimation of the presence of emphysema because of awareness of study design was unlikely.

The small number of NSIP patients in our study needs to be emphasised. NSIP is an uncommon disease, especially with the exclusion of patients with a primary cause and those with clinico-radiological features overlapping with those of hypersensitivity pneumonitis or organizing pneumonia. The NSIP subset examined in the present study consisted of patients presenting with the clinical features of IPF, as studied previously in the U.K. [26] and the U.S.A. [27]. Nevertheless, our observations can now be tested in other cohorts of similar size.

In conclusion, the association of pulmonary emphysema with NSIP (and, to a lesser extent, observations relating to a crazy-paving pattern) provide support for cigarette smoking as a pathogenetic factor in a subset of NSIP patients.

Open Access This article is distributed under the terms of the Creative Commons Attribution Noncommercial License which permits any noncommercial use, distribution, and reproduction in any medium, provided the original author(s) and source are credited.

\section{References}

1. Moon J, du Bois R, Colby TV, Hansell DM, Nicholson AG (1999) Clinical significance of respiratory bronchiolitis on open lung biopsy and its relationship to smoking related interstitial lung disease. Thorax 54:1009-1014
2. Heyneman LE, Ward S, Lynch DA, Remy-Jardin M, Johkoh T, Müller NL (1999) Respiratory bronchiolitis, respiratory bronchiolitis-associated interstitial lung disease, and desquamative interstitial pneumonia: different entities or part of the spectrum of the same disease process? Am J Roentgenol $173: 1617-1622$
3. Taskar VS, Coultas DB (2006) Is idiopathic pulmonary fibrosis an environmental disease? Proc Am Thorac Soc 3:293-298 
4. Cottin V, Nunes H, Brillet PY, Delaval P, Devouassoux G, Tillie-Leblond I, Israel-Biet D, Court-Fortune I, Valeyre D, Cordier JF, Groupe d'Etude et de Recherche sur les Maladies Orphelines Pulmonaires (GERM O P) (2005) Combined pulmonary fibrosis and emphysema: a distinct underrecognised entity. Eur Respir J 26:586-593

5. Craig PJ, Wells AU, Doffman S et al (2004) Desquamative interstitial pneumonia, respiratory bronchiolitis and their relationship to smoking. Histopathology 45:275-282

6. Marten K, Milne D, Nicholson AG, Tenant R, Wells AU, Hansell DM (2005) Smoking-related non-specific interstitial pneumonia: clustering with pulmonary emphysema and CT-based differentiation from idiopathic disease [abstract]. Radiology 217(P):SS0507

7. National Heart Lung and Blood Institute (NHLBI)and World Health Organisation (WHO) (2005) Global Initiative for Chronic Obstructive Lung Disease (GOLD). Global strategy for the diagnosis, management and prevention of COPD. Executive summary (updated 2005). www.goldcopd.org. Accessed 20 Jan 2009

8. Hansell DM, Bankier AA, MacMahon H, McLoud TC, Müller NL, Remy J (2008) Fleischner Society: glossary of terms for thoracic imaging. Radiology 246:697-722

9. Carrington CB, Gaensler EA, Coutu RE, FitzGerald MX, Gupta RG (1978) Natural history and treated course of usual and desquamative interstitial pneumonia. N Engl J Med 298:801809

10. Coultas DB, Zumwalt RE, Black WC, Sobonya RE (1994) The epidemiology of interstitial lung diseases. Am J Respir Crit Care Med 150:967-972
11. Bjoraker JA, Ryu JH, Edwin MK, Myers JL, Tazelaar HD, Schroeder DR, Offort KP (1998) Prognostic significance of histopathologic subjects in idiopathic pulmonary fibrosis. Am J Respir Crit Care Med 157:199-203

12. Turner-Warwick M, Burrows B, Johnson A (1980) Cryptogenic fibrosing alveolitis: clinical features and their influence on survival. Thorax 35:171-180

13. Nagai S, Kitaichi M, Itoh H, Nishimura K, Izumi T, Colby TV (1998) Idiopathic nonspecific interstitial pneumonia/fibrosis: comparison with idiopathic pulmonary fibrosis and bronchiolitis obliterans organizing pneumonia. Eur Respir J 12:1010-1019

14. Daniil ZD, Gilchrist FC, Nicholson AG, Hansell DM, Harris J, Colby TV, du Bois RM (1999) A histologic pattern of nonspecific interstitial pneumonia is associated with a better prognosis than usual interstitial pneumonia in patients with cryptogenic fibrosing alveolitis. Am J Respir Crit Care Med 160:899905

15. Baumgartner KB, Samet JM, Stidley CA, Colby TV, Waldron JA (1997) Cigarette smoking: a risk factor for idiopathic pulmonary fibrosis. Am J Respir Crit Care Med 155:242-248

16. Perez-Padilla R, Salas J, Chapela R, Sanchez M, Carrillo G, Perez R, Sansores R, Gaxiola M, Selman M (1993) Mortality in Mexican patients with chronic pigeon breeder's lung compared with those with usual interstitial pneumonia. Am Rev Respir Dis 148:49-53

17. American Thoracic Society/European Respiratory Society (2002) International multidisciplinary consensus classification of the idiopathic interstitial pneumonias. Am J Respir Crit Care Med 165:277-304

18. MacNee W (2005) Pulmonary and systemic oxidant/antioxidant imbalance in chronic obstructive pulmonary disease. Proc Am Thorac Soc 2:50-60

19. Kinnula VL, Fattman CL, Tan RJ, Oury TD (2005) Oxidative stress in pulmonary fibrosis: a possible role for redox modulatory therapy. Am J Respir Crit Care Med 172:417-422
20. Gauldie J, Kolb M, Ask K, Martin G, Bonniaud P, Warburton D (2006) Smad3 signalling involved in pulmonary fibrosis and emphysema. Proc Am Thorac Soc 3:696-702

21. Ramirez A, Ramadan B, Ritzenthaler JD, Rivera HN, Jones DP, Roman J (2007) Extracellular cysteine/cystine redox potential controls lung fibroblast proliferation and matrix expression through upregulation of transforming growth factor-beta. Am J Physiol Lung Cell Mol Physiol 293(4):L972-L981

22. Antoniou KM, Hansell DM, Rubens MB, Marten K, Desai SR, Siafakas NM, Nicholson AG, du Bois RM, Wells AU (2008) Idiopathic pulmonary fibrosis: outcome in relation to smoking status. Am J Respir Crit Care Med 177:190-194

23. Godwin JD, Müller NL, Takasugi JE (1988) Pulmonary alveolar proteinosis: CT findings. Radiology 169:609-613

24. Johkoh T, Itoh H, Müller NL, Ichikado K, Nakamura H, Ikezoe J, Akira M, Nagareda T (1999) Crazy-paving appearance at thin-section CT: spectrum of disease and pathologic findings. Radiographics 211:155-160

25. Becklake MR, Lalloo U (1990) The "healthy smoker": a phenomenon of health selection? Respiration 57:137144

26. Latsi PI, du Bois RM, Nicholson AG, Colby TV, Bisirtzoglou D, Nikolakopoulou A, Veeraraghavan S, Hansell DM, Wells AU (2003) Fibrotic idiopathic interstitial pneumonia: the prognostic value of longitudinal functional trends. Am J Respir Crit Care Med 168:531-537

27. Flaherty KR, Mumford JA, Murray S, Kazerooni EA, Gross BH, Colby TV, Travis WD, Flint A, Toews GB, Lynch JP 3rd, Martinez FJ (2003) Prognostic implications of physiologic and radiographic changes in idiopathic interstitial pneumonia. Am J Respir Crit Care Med 168:543-548 